\title{
The ninth-mode sectorial oscillation of acoustically levitated drops
}

\author{
YAN ZhenLin, XIE WenJun, GENG DeLu \& WEI BingBo* \\ Department of Applied Physics, Northwestern Polytechnical University, Xi'an 710072, China
}

Received June 4, 2011; accepted July 18, 2011

\begin{abstract}
We report on the ninth-mode sectorial oscillation of acoustically levitated drops excited by actively modulating sound pressure. A numerical computation based on the level set method was performed to model drop shape evolution by solving an incompressible two-phase flow problem. The calculated shapes of the oscillating drop are in good agreement with experimental observations. The relationship between the oscillation frequency and parameters describing the flattened drops is studied both experimentally and numerically. The frequency of the ninth-mode sectorial oscillation decreases with increasing equatorial radius and can be well-described by a modified Rayleigh equation.
\end{abstract}

acoustic levitation, drop dynamics, sectorial oscillation, level set method

Citation: Yan Z L, Xie W J, Geng D L, et al. The ninth-mode sectorial oscillation of acoustically levitated drops. Chinese Sci Bull, 2011, 56: 3284-3288, doi: $10.1007 / \mathrm{s} 11434-011-4698-2$

Drop oscillation has been attracting considerable attention in various fields for over a century. In the last few decades, a series of studies have been performed to explore the oscillatory dynamics of liquid drops with both experimental and numerical techniques [1-10]. Many experimental studies involving axisymmetric oscillations were performed using electromagnetic levitation, acoustic levitation, electrostatic levitation and aerodynamic levitation in recent years $[4,6-8,11]$. However, only few studies have been attempted on non-axisymmetric oscillations of an isolated drop [12-14]. Understanding the nature of the non-axisymmetric oscillations is desirable, not only from a scientific viewpoint but also for practical aspects related to material processing under containerless conditions.

The suspension of liquid drops provides a preferable boundary condition for the investigations of various free surface phenomena. Acoustic levitation is an important containerless processing technique for in situ investigations of free-drop dynamics that require special physical environments [15-18]. It has the particular advantage of not being constrained by electromagnetic properties of the samples.

Sectorial oscillations are a non-axisymmetric type of

*Corresponding author (email: bbwei@nwpu.edu.cn) oscillation and can be characterized by either top-view or bottom-view images. Thus far, single-mode sectorial oscillations of acoustically levitated water drops have been observed up to the eighth mode. In this work, we report the ninth-mode sectorial oscillation obtained using an active modulation method. A numerical computation incorporating level set method is employed to model the observed drop shape evolution. Both experimental measurement and numerical prediction are used to investigate the relationship between the oscillation frequency and parameters describing the flattened drops.

\section{Experimental method}

The experiments are performed using a single-axis acoustic levitator, which is actuated by a piezoelectric (PZT) transducer at a frequency of $22 \mathrm{kHz}$. In the experiments, the acoustic radiation force suspends a water drop with diameter of $5 \mathrm{~mm}$. To excite the levitated water drop into an oscillating state, we actively modulated the electric voltage $V$ applied to the PZT transducer. The form of this modulation is $V=V_{0}\left(1+\eta \cos \omega_{\mathrm{m}} t\right) \cos \omega_{\mathrm{ac}} t$, where $\omega_{\mathrm{m}}$ and $\omega_{\mathrm{ac}}$ are the angular frequencies of the modulation signal and the ultrasound, 
respectively, and $\eta$ the ratio of the modulation amplitude to the initial electric voltage $V_{0}$. To flatten the levitated drop to a higher extent, a plane reflector replaces the conventional curved reflector to favorably excite the high-order sectorial oscillations. The bottom-view images of the oscillating drop are recorded by a Redlake HG $100 \mathrm{~K}$ high speed CCD camera at a rate of $5000 \mathrm{fps}$. The oscillation frequency is obtained by analyzing the recorded images.

\section{Results and discussion}

\subsection{Experimental observation}

Panels (a1)-(a5) in Figure 1 show a typical sequence of the nine-lobed oscillation. The sequence records half a cycle of oscillation and the corresponding oscillation frequency is $240.4 \mathrm{~Hz}$. The oscillation amplitude is determined as $0.21 \mathrm{~mm}$. The experimentally measured oscillation frequency is exactly half the corresponding modulation frequency, which is $487.5 \mathrm{~Hz}$. During the oscillation, the fluid inside the water drop is transferred from the lobe region to the valley between two neighboring lobes. Nine equally-spaced lobes alternately stretch out and contract back.

For the ninth-mode sectorial oscillation of a deformed drop, the shape evolution can be expressed as $r(\theta, \varphi, t)=r_{0}(\theta)\left\{1+\xi_{0} \sin ^{9} \theta \cos \left[9\left(\varphi+\varphi_{0}\right)\right] \cos \omega t\right\}$,

where $r_{0}(\theta)$ denotes the equilibrium shape of the initially flattened drop, $\xi_{0}$ the relative oscillation amplitude, $\omega$ the oscillation angular frequency, $\theta$ and $\varphi$ the polar angle and azimuthal angle respectively, and $\varphi_{0}$ the initial phase of the azimuthal angle. By inserting $\theta=\pi / 2$ into eq. (1), the equatorial flange of the drop is given by

$$
r(\varphi, t)=R_{\mathrm{e}}\left\{1+\xi \cos \left[9\left(\varphi+\varphi_{0}\right)\right]\right\}
$$

where $R_{\mathrm{e}}$ is the equatorial radius and $\xi=\xi_{0} \cos (\omega t)$ the transient deviation amplitude.

To further identify the oscillation mode, five selected images corresponding to the phases of $0, \pi / 2, \pi, 3 \pi / 2$, and $2 \pi$ in one cycle of evolution are compared with those theoretically predicted by eq. (2), as shown in Figure 2. For the sake of clarity, the extracted peripheries of the oscillating drop are presented as dots and the theoretical results are plotted as solid lines in Figure 2. It is clear that experiment and theory agree with each other. This gives further evidence that the oscillation observed in our experiments is the ninth-mode sectorial oscillation.

The modulation of sound pressure leads to a periodical

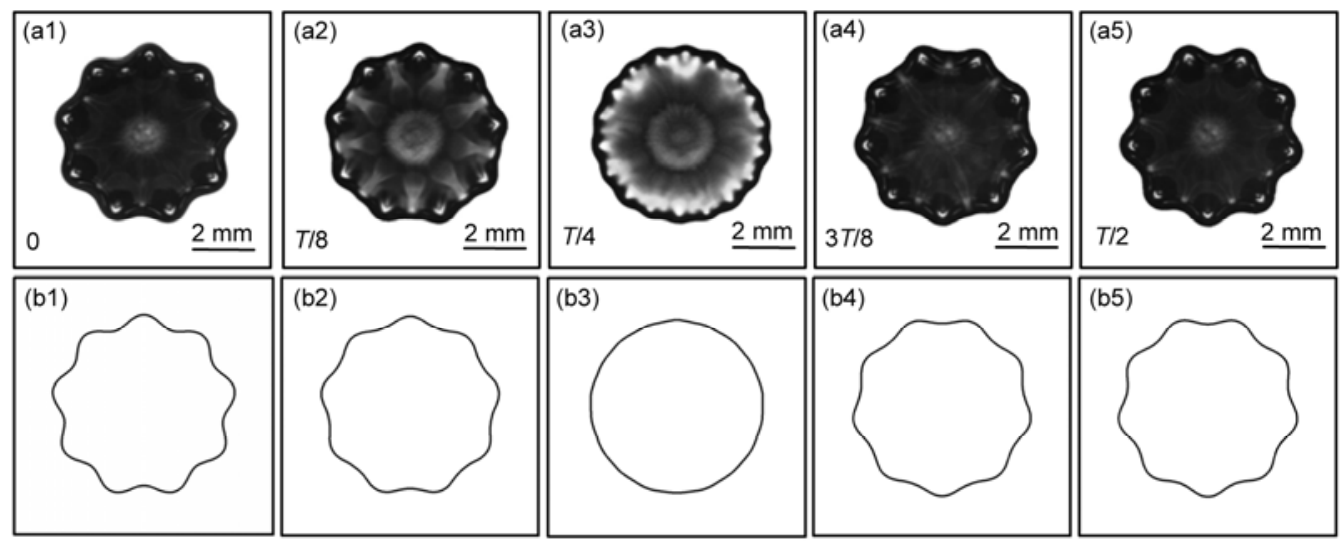

Figure 1 Snapshots of a water drop undergoing the ninth-mode sectorial oscillation. (a1)-(a5) Experimental observation; (b1)-(b5) numerical predictions of the water drop envelop.

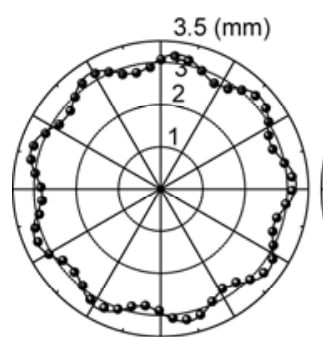

(a)

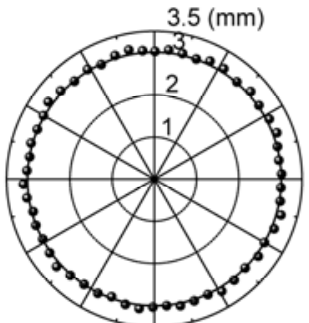

(b)

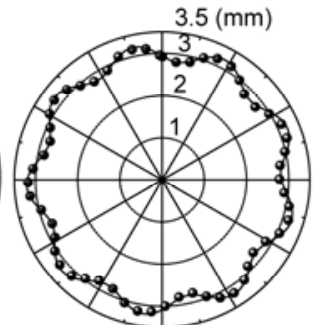

(c)

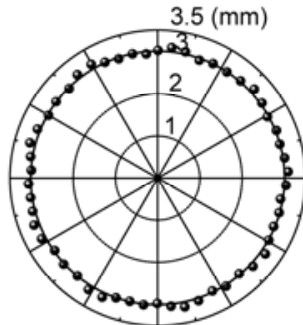

(d)

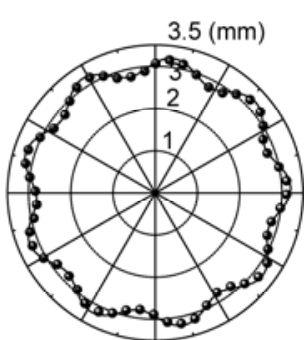

(e)

Figure 2 Time evolution of equatorial contour for one cycle. (a) Phase $\omega t=0$; (b) $\omega t=\pi / 2$; (c) $\omega t=\pi$; (d) $\omega t=3 \pi / 2$; (e) $\omega t=2 \pi$. 
fluctuation in the extent of distortion for the levitated drop. A forced axisymmetric oscillation due to this modulation is usually excited in our experiments. For a levitated drop undergoing this kind of oscillation, its equatorial radius varies according to $R_{\mathrm{e}}=R_{\mathrm{e} 0}\left[1+A_{0} \cos \left(\omega_{\mathrm{m}} t\right)\right]$, or $\delta R_{\mathrm{e}} / R_{\mathrm{e} 0}=A_{0} \cos \left(\omega_{\mathrm{m}} t\right)$. Here, $R_{\mathrm{e} 0}$ is the equatorial radius of the levitated drop before modulation and $A_{0}$ the maximum deviation of the equatorial radius. Figure 3 shows the time evolution of $\xi$ and $\delta R_{\mathrm{e} 0} / R_{\mathrm{e} 0}$ within three oscillating cycles. In Figure 3 , the dots and open circles are the experimental data of $\xi$ and $\delta R_{\mathrm{e} 0} / R_{\mathrm{e} 0}$ obtained from the recorded images. The solid line and dashed lines correspond to the calculated results from equations $\xi=$ $\xi_{0} \cos (\omega t)$ and $\delta R_{\mathrm{e}} / R_{\mathrm{e} 0}=A_{0} \cos \left(\omega_{\mathrm{m}} t\right)$. Here, the relative oscillation amplitude $\xi_{0}$ and the maximum deviation of equatorial radius $A_{0}$ are 0.07 and 0.01 , respectively. Clearly, the theoretical modeling is in close agreement with the experimental measurements. In Figure 3, the frequency of the axisymmetric oscillation is equal to the modulation frequency $\omega_{\mathrm{m}}$ and that of the sectorial oscillation is half of $\omega_{\mathrm{m}}$. We also observed this phenomenon in our previous experiments [19], demonstrating it to be a parametric resonance. The ninth-mode sectorial oscillation is also parametrically excited by the periodical perturbation of the equatorial radius due to the fluctuation of the electric voltage applied to the ultrasound transducer.

\subsection{Sectorial oscillation frequency}

The experimental results on free decay of a ninth-mode sectorial oscillation are given in Figure 4. The transient amplitudes were obtained from the digitized drop images and are plotted as a function of time in Figure 4(a). These amplitudes can be fitted with the exponential function $0.017 \times$ $\exp [-(t-0.1) / 0.075)]$. In this example, the corresponding oscillation frequency is $218.5 \mathrm{~Hz}$. Figure 4(b) displays the frequency spectrum, obtained by FFT, of the levitated drop. Clearly, only one frequency peak appears. The oscillation

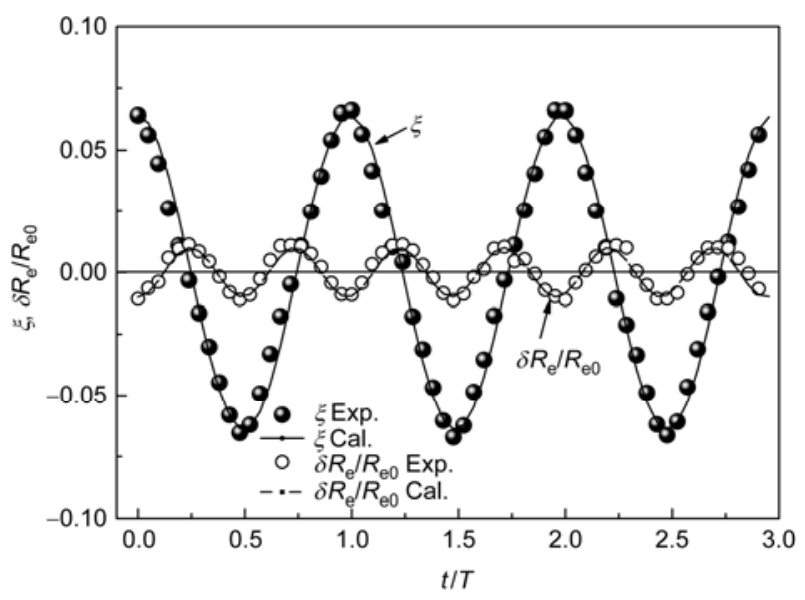

Figure 3 Evolution of the transient amplitude for an acoustically levitated water drop over three oscillating cycles.

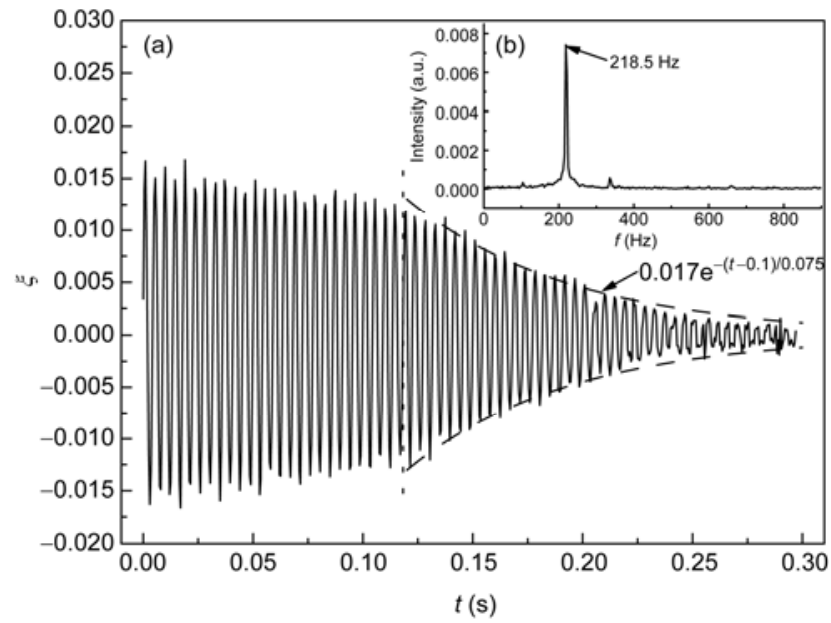

Figure 4 Decay process of an oscillating drop. (a) Time variation of the amplitude in free decay phase; (b) oscillation spectrum of the levitated drop from FFT.

frequency does not change during the free-decay process, indicating that the drop oscillates at its natural frequency. The modulation of sound pressure only provides appropriate energy to counteract the viscosity dissipation of the drop. This characteristic of sectorial oscillations provides a specific method to measure the natural frequency of the flattened drops.

The natural frequency of a free drop undergoing smallamplitude oscillations is given by the Rayleigh equation [20]. The static drop deformation associated with acoustic levitation has been determined to induce a decrease in the resonant frequency of small-amplitude shape oscillation. In our experiments, water drops were severely flattened into a concave shape by the acoustic radiation pressure. The natural frequency in the present experiments significantly deviates from the value predicted by the Rayleigh equation. According to the evidence to date, a detailed theoretical analysis to predict the natural frequency of drastically-deformed liquid drops is not yet available.

The relationship between the natural frequency and the parameters of deformed drops was further analyzed. The sectorial oscillation frequency was measured as a function of the initial equatorial radii $R_{\mathrm{e}}$, as shown in Figure 5, where it decreases with increasing equatorial radius. The dependence of the measured oscillation frequency on the equatorial radius is well-described by a modified expression of the Rayleigh equation where the equatorial radius $R_{\mathrm{e}}$ is replaced by the equivalent spherical radius:

$$
f_{l}=\frac{1}{2 \pi} \sqrt{\frac{\sigma}{\rho R_{\mathrm{e}}^{3}} l(l-1)(l+2)},
$$

where $\sigma$ and $\rho$ are the surface tension and density of the drop respectively, and $l$ is the mode of oscillation. The empirically predicted results using eq. (3) are found to be slightly lower than experimental values. The difference 


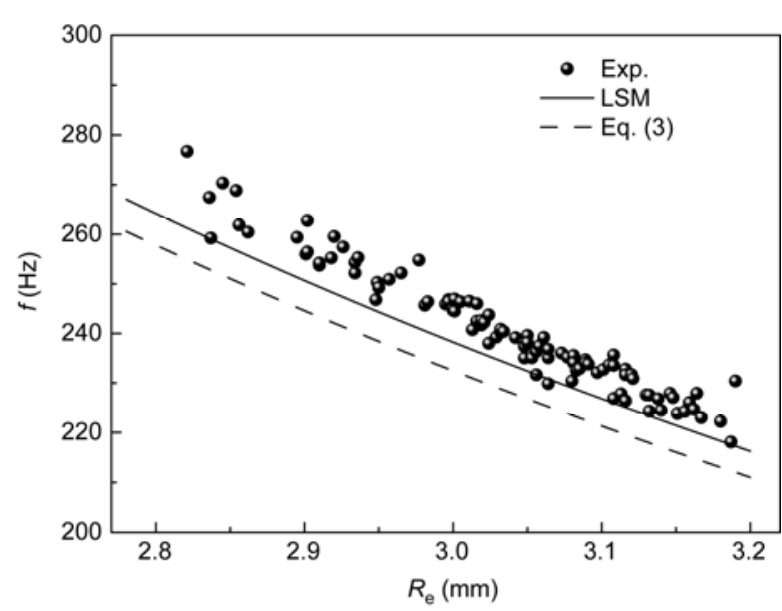

Figure 5 Dependence of oscillation frequency on equatorial radius.

between experimental and predicted results is to within $5 \%$.

\subsection{Numerical analysis}

To obtain a brief overview of the physics of the ninth-mode sectorial oscillation, a numerical computation was performed to simulate the shape evolution of free drops oscillating in air. Since the fluid velocity is much less than the speed of sound, we can approximate the fluids as being incompressible. To simplify the problem, the acoustic radiation force was assumed to only suspend the water drop and had no influence on the oscillation process. Therefore, in this simulation, the sound field and gravity were not taken into consideration. During sectorial oscillation, the levitated water drop was significantly fattened to an aspect ratio of 6 . In addition, fluid flow only occurs in the horizontal plane [21], and fluid velocities in the vertical direction are zero. Hence, it is reasonable to simplify the simulation to just two dimensions.

In the calculation of the two-phase flow with free boundary, a key problem is the interface tracking. Instead of explicitly tracking, a level set method (LSM) [22] is used to implicitly capture the water/air interface. Here we briefly illustrate the outline of the level set method. The level set function $\Phi$ is defined as $\Phi=0$ in the liquid region, $\Phi=1$ in the gas region, and $\Phi=0.5$ at the interface. The air/water interface is identified at points where $\Phi=0.5$. The evolution equation of $\Phi$ is given by

$$
\frac{\partial \Phi}{\partial t}+\boldsymbol{u} \cdot \nabla \Phi=0
$$

where $\boldsymbol{u}$ is the fluid velocity vector. The incompressible Navier-Stokes equations in dimensionless form are

$$
\begin{gathered}
\nabla \cdot \boldsymbol{u}=0 \\
\frac{\partial \boldsymbol{u}}{\partial t}+(\boldsymbol{u} \cdot \nabla) \boldsymbol{u}=-\frac{\nabla p}{\rho}+\frac{\nabla \cdot\left\{\mu\left[\nabla \boldsymbol{u}+(\nabla \boldsymbol{u})^{\mathrm{T}}\right]\right\}}{\rho R e}-\frac{\kappa \nabla \Phi}{\rho W e}
\end{gathered}
$$

where $\rho, p, \mu$ and $\kappa$ are the density, pressure, viscosity and curvature of the interface, respectively. To avoid numerical instabilities, the density $\rho$ and viscosity $\mu$ should be smoothed at the interface by a smeared out Heaviside function. The surface tension stress is transformed to a volume force spread over a few layers of cells with a diffuse interface model [23]. The Reynolds number $R e=\rho_{0} U L / \mu_{0}$ has value 896 , and the Weber number $W e=\rho_{0} U^{2} L / \sigma$ is 3.69. Here, the reference density $\rho_{0}=0.998 \times 10^{3} \mathrm{~kg} / \mathrm{m}^{3}$ and reference viscosity $\mu_{0}=1.002 \times 10^{-3} \mathrm{~Pa}$ s are set to those of water at $293 \mathrm{~K}$. The reference length $L=3.0 \times 10^{-3} \mathrm{~m}$ is chosen as the equatorial radius of the drop, and the reference velocity of $U=0.3 \mathrm{~m} / \mathrm{s}$ equals the maximum fluid velocity in the oscillating drop. The surface tension of water is $\sigma=0.073 \mathrm{~N} / \mathrm{m}$. The waterto-air density and viscosity ratios are about $830: 1$ and $60: 1$, respectively.

To numerically solve the Navier-Stokes equations using the level set method over fixed grids, the staggered mesh was used for spatial discretization. The convective terms were discretized using second-order upwind scheme. The viscous stress tensor and the curvature were discretized with the central difference scheme. The time integration was performed by second-order Runge-Kutta method. Due to the convective terms, viscous terms and surface tension terms, the time step must obey the Courant-Friedrichs-Lewy (CFL) conditions. Pressure and velocity fields were solved numerically using the extensions of the Marker and Cell (MAC) method together with the conservative total variation diminishing (TVD) advection scheme. The initial configuration of the drop was set to the shape of the oscillating drop at maximum deviation amplitude of 0.07 . The computational fluid domain was $4 \times 4$ and the grid size $320 \times 320$, where a free-slip condition is assumed at the domain boundaries.

Panels (b1)-(b5) in Figure 1 depict the evolution of the shape oscillations from the above numerical computation. The result is in good agreement with the experimental results. The numerical computation indicates that the surface tension stress resulting from curvature change is the dominant restoring force that sustains the oscillation. The modulation of the ultrasound field only helps to counteract the viscosity dissipation of energy. In addition, a related computation is also conducted to estimate the frequency for the different equatorial radii. The solid line in Figure 5 represents the calculated results. The simulated results are slightly lower than the experimental measurements and slightly higher than the empirical predications given by eq. (3). Overall, the numerical computation provides an effective modeling of frequencies of this ninth-mode sectorial oscillation.

\section{Conclusions}

The ninth-mode sectorial oscillation of acoustically levitated drops was successfully excited by actively modulating 
the sound pressure. The oscillation frequency decreased with increasing equatorial radius and was well-described by our modified Rayleigh equation. Numerical analysis was performed to predict the shape evolution of the oscillating drop using a conservative level set method to solve the corresponding incompressible two-phase flow problem. The simulated shapes were found to agree well with experimental observations. Both experimental and numerical methods were used to investigate the dependence of the oscillation frequency on parameters describing flattened drops. Numerical results indicate that the computational simulation provided satisfactory results in predicting the frequencies of these sectorial oscillations.

The authors thank Dr. H. P. Wang, Mr. J. Chang and Mr. K. Zhou for beneficial discussions. This work was supported by the National Natural Science Foundation of China (50971105, 51071126).

1 Lai M F, Lee C P, Liao C N, et al. Oscillation spectrums and beat phenomenon of a water droplet driven by electrowetting. Appl Phys Lett, 2009, 94: 154102

2 Oh J M, Ko S H, Kang K H. Shape oscillation of a drop in ac electrowetting. Langmuir, 2008, 24: 8379-8386

3 Saylor J R, Jones B K. The existence of vortices in the wakes of simulated raindrops. Phys Fluids, 2005, 17: 031706

4 Ozawa S, Adachi M, Morohoshi K, et al. The influence of temporal phase difference of $m= \pm 2$ oscillations on surface frequency analysis. J Appl Phys, 2009, 106: 034907

5 Moon J H, Kang B H, Kim H Y. The lowest oscillation mode of a pendant drop. Phys Fluids, 2006, 18: 021702

6 Beaugnon E, Fabregue D, Billy D, et al. Dynamics of magnetically levitated droplets. Physica B, 2001, 294: 715-720

7 Yarin A L, Weiss D A, Brenn G, et al. Acoustically levitated drops: Drop oscillation and break-up driven by ultrasound modulation. Int $\mathbf{J}$ Multiphase Flow, 2002, 28: 887-910
8 Trinh E H, Holt R G, Thiessen D B. The dynamics of ultrasonically levitated drops in an electric field. Phys Fluids, 1996, 8: 43-61

9 Lopez H, Sigalotti L D G. Oscillation of viscous drops with smoothed particle hydrodynamics. Phys Rev E, 2006, 73: 051201

10 Feng Z C, Su Y H. Numerical simulations of the translational and shape oscillations of a liquid drop in an acoustic field. Phys Fluids, 1997, 9: 519-530

11 Hervieu E, Coutris N, Boichon C. Oscillations of a drop in aerodynamic levitation. Nucl Eng Des, 2001, 204: 167-175

12 Hill R J A, Eaves L. Nonaxisymmetric shapes of a magnetically levitated and spinning water droplet. Phys Rev Lett, 2008, 101: 234501

13 Noblin X, Buguin A, Brochard-Wyart F. Triplon modes of puddles. Phys Rev Lett, 2005, 94: 166102

14 Azuma H, Yoshihara S. Three-dimensional large-amplitude drop oscillations: experiments and theoretical analysis. J Fluid Mech, 1999, 393: 309-332

15 Yan N, Geng D L, Hong Z Y, et al. Rapid solidification of acoustically levitated Al-Cu-Si eutectic alloy under laser irradiation. Chinese Sci Bull, 2011, 56: 912-918

16 Hong Z Y, Lü Y J, Xie W J, et al. The liquid phase separation of $\mathrm{Bi}-\mathrm{Ga}$ hypermonotectic alloy under acoustic levitation condition. Chinese Sci Bull, 2007, 52: 1446-1450

17 Lü Y J, Cao C D, Wei B B. High undercooling of bulk water during acoustic levitation. Sci China Ser G-Phys Mech Astron, 2003, 46: 259-267

18 Shen C L, Xie W J, Wei B B. Digital image processing of sectorial oscillations for acoustically levitated drops and surface tension measurement. Sci China Phys Mech Astron, 2010, 53: 2260-2265

19 Shen C L, Xie W J, Wei B. Parametrically excited sectorial oscillation of liquid drops floating in ultrasound. Phys Rev E, 2010, 81: 046305

20 Rayleigh L. On the capillary phenomena of jets. Proc R Soc London, 1879, 29: 71-97

21 Shen C L, Xie W J, Wei B. Internal flow of acoustically levitated drops undergoing sectorial oscillations. Phys Lett A, 2010, 374: 4045-4048

22 Olsson E, Kreiss G. A conservative level set method for two phase flow. J Comput Phys, 2005, 210: 225-246

23 Brackbill J U, Kothe D B, Zemach C. A continuum method for modeling surface tension. J Comput Phys, 1992, 100: 335-353

Open Access This article is distributed under the terms of the Creative Commons Attribution License which permits any use, distribution, and reproduction in any medium, provided the original author(s) and source are credited. 